\title{
BARIUM AND IRON ABUNDANCES IN RED GIANTS
}

\author{
J. L. Fernández-Villacañas, M. Rego, ANd M. Cornide \\ Departamento de Astrofísica, Facultad de Ciencias Físicas, Universidad Complutense, 28040 Madrid, Spain \\ Received 10 August 1989; revised 20 December 1989
}

\begin{abstract}
An intermediate-dispersion abundance analysis has been carried out on a sample of 21 barium and 14 comparison stars. The excess of barium over iron has been used as the most representative indicator of peculiarity. These excesses are higher in the peculiar stars than in the nonpeculiar stars. Particularly interesting is the case of $\mathrm{HD} 67447$, included in the comparison stars, with an excess $[\mathrm{Ba} / \mathrm{Fe}]=1.61$, probably a new barium star. A trend indicating a possible anticorrelation between barium overabundance and metallicity favors the suggestion that the "barium strong" group is older than the "barium weak" one.
\end{abstract}

\section{INTRODUCTION}

Barium stars represent an important group within lowmass cool giants and are good examples of the possible performance of the s process, which leads to the formation of heavy elements such as barium and strontium. Observation of elements of this kind in detailed analyses of the stars under study has led, in most cases, to the determination of overabundance of barium and rare earths, as well as $\mathrm{Y}$ and $\mathrm{Zr}$.

This kind of giant was first acknowledged as a class by Bidelman and Keenan (1951), who considered the enhancements that the $\mathrm{Ba}$ II, Sr II, and some carbon molecules presented. Since then, a series of low-resolution studies has been carried out dealing with the characteristics of these stars. Among them stands out the work by Lü et al. (1983), based on the measurement of the Ba II $\lambda 4554 \AA$ line. He classified these stars on the basis of a qualitative index of barium line strength, previously introduced by Warner (1965), in a scale ranging from 1 to 5 on low-dispersion spectrograms. Other works in this field are those by McConnell et al. (1972) and Williams (1975). The latter introduced an index, $R(B a)$, which measures the peculiarity of these stars from narrowband photometry studies and determines barium abundances using models and spectral synthesis as tools.

Barium stars differ in luminosity class among themselves. The classification according to this parameter is not clear in most cases, making it rather difficult to discern the effects of luminosity from those typical of the internal chemical composition. A similar, though less conspicuous, problem occurs with spectral types, whose determination sometimes varies depending on the source.

Former studies lead us to the cataloging of these stars into several types (mild, marginal, certain, etc.), but the truth is that the existing classifications do not represent a definite characterization, which can be determined only through detailed abundance calculations. So, low-dispersion analyses have given way to high-resolution studies, where the abundance of barium and other elements (including a number of s-process elements) is determined. These results are based on very high-dispersion spectrograms, and for this reason they are applied, to this date, to a rather restricted number of barium stars. From these works we can underline those by Tomkin and Lambert (1983) and Kovacs (1983, 1985). Both sources find enhancements in the s-process elements as well as in the $\mathrm{C} / \mathrm{O}$ ratio when compared with nonpeculiar stars. Solid-state detectors (Digicon and Reticon) were used in these works, allowing much higher resolution and precision than those obtained in previous studies.
In the present work, an intermediate analysis concerning the aforementioned has been carried out. The spectra have intermediate dispersion so that the resolution is better than that of low-dispersion studies. At the same time, this allows us to include a higher number of stars in lieu of the better quality of high-resolution studies. In this sense, we have approached the analysis of iron and barium abundances for a sample of comparison stars and barium stars. The red part of the optical spectrum has been chosen as the basis for our work; the blanketing effects are not as important there as in the blue spectral region. More importantly, this has been a poorly studied region for these kinds of stars.

\section{OBSERVATIONS AND REDUCTION}

The sample observed contains 21 barium stars and 14 comparison stars of the same spectral type and luminosity class. Their most important data are listed in Table I. The spectral types range between $\mathrm{G} 7$ and $\mathrm{M} 0$ and the luminosity classes between III and I. These have been taken from Hoffleit and Jaschek (1982) and Keenan (1983, 1984, 1985). Trigonometric parallaxes are those from Hoffleit and Jaschek (1982). The barium peculiarity indicator is that of Keenan (1983) and Lü et al. (1983). It is to be remarked that, except for HR 774 and $56 \mathrm{Peg}$, the values of the barium indices are low. Tables II (a) and II(b) show the most important photometric data of the sample of stars which have been used in the elaboration of later results. The indices in the Johnson system have been obtained from Hoffleit and Jaschek (1982). DDO photometry has been taken from McClure (1976) and McClure and Forrester (1981).

The observations were carried out with the $1.52 \mathrm{~m}$ telescope coudé spectrograph of the Observatorio Astronómico Nacional at Calar Alto (Almería) during two observing periods in 1985 and 1986. 098 and IIIa-F photographic emulsions were used as the detector. The spectra were obtained at a reciprocal dispersion of $21 \AA / \mathrm{mm}(0.4 \AA$ resolution $)$ in the spectral region 5800-6000 $\AA$, which includes the three Ba II lines analyzed in our work. Exposure times ranged from several minutes for the brightest stars in the sample to $1-2 \mathrm{hr}$ for the faintest.

Calibration was performed with the help of an ETA spectrograph, as well as with comparison star spectra of known energy distribution. The comparison stars selected for that purpose were Vega ( $\alpha$ Lyr) and Arcturus ( $\alpha$ Boo). The spectra were scanned with a PDS microdensitometer.

Finally, equivalent widths for 16 isolated $\mathrm{Fe} I$ lines distributed along the spectrum and three spectral features contain- 
TABLE I. Basic data. Columns 1 and 2: HD and HR identifications; column 3: star name; column 4: spectral type and luminosity class; column 5: Keenan peculiarity index; columns 6 and 7: right ascension and declination (1950.0); column 8: trigonometric parallax ("); column 9: apparent visual magnitude; column 10: number of observations.

\begin{tabular}{|c|c|c|c|c|c|c|c|c|c|c|c|c|c|c|}
\hline \multirow{2}{*}{$\begin{array}{c}\text { HD } \\
\text { Ba star }\end{array}$} & \multirow[t]{2}{*}{ HR } & \multicolumn{2}{|c|}{ Name } & Sp.T. & \multirow[t]{2}{*}{$\mathrm{Ba}$} & \multicolumn{3}{|c|}{ R.A. } & \multicolumn{3}{|c|}{ DEC. } & \multirow[t]{2}{*}{ PAR. } & \multirow[t]{2}{*}{$\mathrm{m}_{\mathrm{v}}$} & \multirow[t]{2}{*}{$\mathrm{N}$} \\
\hline & & & & & & & & & & & & & & \\
\hline 5395 & 265 & $v^{2}$ & Cas & G8.5 IIIb & 0.3 & 0 & 53 & 40 & 58 & 54 & 41 & 0.051 & 4.66 & 3 \\
\hline 13520 & 643 & 60 & And & $\mathrm{K} 3.5 \mathrm{III}$ & 0.5 & 2 & 10 & 04 & 43 & 59 & 53 & & 4.84 & 2 \\
\hline 13611 & 649 & 65 & cet & G6 II-III & 0.3 & 2 & 10 & 20 & 8 & 36 & 47 & 0.022 & 4.37 & 3 \\
\hline 16458 & 774 & & & G8p & 3.0 & 2 & 40 & 25 & 81 & 14 & 22 & 13 & 5.76 & 1 \\
\hline 30834 & 1551 & 2 & Aur & $\mathrm{K} 2.5$ IIIb & 0.4 & 4 & 49 & 16 & 36 & 37 & 14 & & 4.77 & 2 \\
\hline 39003 & 2012 & 32 & Aur & G9.5 III & 0.2 & 5 & 48 & 01 & 39 & 08 & 10 & .017 & 3.97 & 2 \\
\hline 43232 & 2227 & $\gamma$ & Mon & $\mathrm{K} 1.5 \mathrm{III}$ & 0.3 & 6 & 12 & 25 & -6 & 15 & 29 & 13 & 3.97 & 2 \\
\hline 59294 & 2864 & 6 & $\mathrm{CMi}$ & $\mathrm{K} 1+\mathrm{III}$ & 0.4 & 7 & 27 & 00 & 12 & 06 & 41 & 0.029 & 4.53 & 3 \\
\hline 69267 & 3249 & $\beta$ & Cnc & K4 III & 0.5 & 8 & 13 & 48 & 9 & 20 & 27 & 0.012 & 3.52 & 4 \\
\hline 74739 & 3475 & l & Cnc & G7.5 IIIa & 0.1 & 8 & 43 & 40 & 28 & 56 & 39 & 7 & 4.20 & 3 \\
\hline 82308 & 3773 & $\lambda$ & Leo & $\mathrm{K} 4.5 \mathrm{III}$ & 0.3 & 9 & 28 & 52 & 23 & 11 & 22 & 20 & 4.31 & 2 \\
\hline 83618 & 3845 & j & Hya & $\mathrm{K} 2.5$ III-IIIb & 0.3 & 9 & 37 & 18 & -0 & 54 & 53 & 0.026 & 3.89 & 3 \\
\hline 95345 & 4291 & 58 & Leo & K1 III & $1 *$ & 10 & 57 & 58 & 3 & 53 & 10 & 0.007 & 4.83 & 2 \\
\hline 95578 & 4299 & 61 & Leo & MO III & 0.2 & 10 & 59 & 17 & -2 & 12 & 55 & & 4.74 & 1 \\
\hline 98262 & 4377 & $v$ & UMa & K3- III & 0.3 & 11 & 15 & 46 & 33 & 22 & 02 & 0.020 & 3.70 & 3 \\
\hline 98839 & 4392 & 56 & UMa & G7.5 IIIa & 0.3 & 11 & 21 & 50 & 43 & 45 & 26 & 0.002 & 5.10 & 1 \\
\hline 119228 & 5154 & 83 & UMa & M2 IIIab & 1.0 & 13 & 38 & 50 & 54 & 56 & 03 & 0.014 & 4.66 & 1 \\
\hline 133208 & 5602 & $\beta$ & Boo & G8 IIIa & 0.4 & 15 & 00 & 04 & 40 & 35 & 13 & 0.037 & 3.49 & 1 \\
\hline 202109 & $8115^{\circ}$ & 64 & Cyg & G8 III-IIIa & 0.6 & 21 & 10 & 48 & 30 & 01 & 15 & 0.027 & 3.40 & 1 \\
\hline 206778 & 8308 & $\varepsilon$ & Peg & $\mathrm{K} 2 \mathrm{Ib}$ & & 21 & 41 & 44 & 9 & 38 & 42 & 0.006 & 2.38 & 1 \\
\hline 218356 & 8796 & 56 & Peg & G8 Ib & $2 \star$ & 23 & 04 & 40 & 25 & 11 & 53 & 0.006 & 4.77 & 1 \\
\hline
\end{tabular}

(*) barium peculiarity taken from Lü et al. (1983)

\begin{tabular}{|c|c|c|c|c|c|c|c|c|c|c|c|c|c|}
\hline \multicolumn{14}{|c|}{ Comparison stars } \\
\hline 3627 & 165 & $\delta$ & And & K3 III & 0 & 36 & 39 & 30 & 35 & 16 & 0.028 & 3.27 & 1 \\
\hline 12533 & 603 & 57 & And & $\mathrm{K} 3-\mathrm{IIb}$ & 2 & 00 & 50 & 42 & 05 & 31 & 0.013 & 2.26 & 2 \\
\hline 27371 & 1346 & $\gamma$ & Tau & Ko- IIIab & 4 & 16 & 56 & 15 & 30 & 29 & 0.028 & 3.65 & $\overline{1}$ \\
\hline 28305 & 1409 & $\varepsilon$ & Tau & G9.5 III & 4 & 25 & 42 & 19 & 04 & 16 & 0.020 & 3.53 & $\overline{1}$ \\
\hline 29139 & 1457 & $\alpha$ & Tau & $\mathrm{K} 5+\mathrm{III}$ & 4 & 33 & 03 & 16 & 24 & 38 & 0.054 & 0.85 & 7 \\
\hline 62345 & 2985 & $k$ & Gem & G8 IIIa & 7 & 41 & 26 & 24 & 31 & 10 & 0.026 & 3.57 & 1 \\
\hline 67447 & 3182 & & & G7+ II & 8 & 07 & 52 & 68 & 37 & 26 & 0.021 & 5.50 & 2 \\
\hline 76294 & 3547 & 16 & Hya & G9 II-III & 8 & 52 & 45 & 6 & 08 & 13 & 0.035 & 3.11 & 1 \\
\hline 77912 & 3612 & & & G7 Ib-II & 9 & 03 & 21 & 38 & 39 & 12 & 0.021 & 4.70 & 2 \\
\hline 124897 & 5340 & $\alpha$ & Boo & $\mathrm{K} 1+\mathrm{III}$ & 14 & 13 & 23 & 19 & 26 & 31 & 0.097 & -0.04 & 2 \\
\hline 129312 & 5480 & 31 & Boo & G7+ III & 14 & 39 & 11 & 8 & 22 & 28 & 0.011 & 4.86 & 1 \\
\hline 163770 & 6695 & $\hat{\theta}$ & Her & K1 IIa & 17 & 54 & 32 & 37 & 15 & 22 & 0.002 & 3.86 & 1 \\
\hline 205435 & 8252 & م & Cyg & G8 III & 21 & 32 & 06 & 45 & 22 & 12 & 0.002 & 4.02 & 1 \\
\hline 210807 & 8468 & 24 & Cep & G7 II-III & 22 & 08 & 51 & 72 & 05 & 41 & 0.017 & 4.79 & , \\
\hline
\end{tabular}

ing $\mathrm{Ba}$ II lines were measured for the 35 stars in the sample. These are given in Tables III (a)-III(e). The Ba II $\lambda 5853.68$ $\AA$ Aine appears blended with the Fe $\mathrm{L} \lambda \lambda \mathbf{5 8 5 2 . 2 2}$ and 5855.08 $\AA$ lines. The Ba II $\lambda 6141.72 \AA$ seems to be blended with a Fe I line, though, in this case the $\mathrm{Ba}$ II line is the main contributor to the blend. The third spectral feature contains both the Ba II $\lambda 6496.90 \AA$ and the $\mathrm{Fe}$ I $\lambda 6496.47 \AA$ lines. Line wavelengths were taken from Moore et al. (1966).

\section{ANALYSIS: THE SPECTRAL SYNTHESIS}

Due to the resolution of our spectra, the Ba II lines appeared to be blended, mainly with $\mathrm{Fe}$ I lines. To solve this problem the whole area of the blend was measured, and with the help of a spectral synthesis program the barium abundance could be derived once the iron abundance was known.

The spectral synthesis (see Cornide and Rego 1985 for details) was performed taking as the starting point the choice of a model atmosphere from the grid calculated by Gustafsson and his colleagues (kindly provided by Dr. Gustafsson in a private communication). No interpolation was carried out among different models because the uncertainties introduced in the measuring of the equivalent widths were higher than the fluctuations resulting from assigning two or more close models. Therefore, each star was assigned the model whose parameters were the closest to those previously calculated by the different methods described below. Hydrostatic equilibrium and ionization equations were solved in the usual way, taking into account the line-transfer equation in the LTE hypothesis in order to obtain equivalent widths for a set of different values of abundance.

Since barium stars belong to the old disk population (McClure 1984), it seems reasonable that their metallicities would not be very different from the solar abundance. Therefore, that value was generally adopted for our sample of barium and comparison stars; only for those stars with previously published metallicities was that parameter modified in the sense of assigning lower values than in the solar case. This was done according to the following criterion: if the iron abundance from Morel et al. (1976) and Williams (1975) was negative for a certain star, the Gustafsson models with metallicity -0.25 dex with respect to the solar case were used. 
The calculation of a spectral synthesis requires reliable values of the excitation potentials and oscillator strengths. After a comparative study of observed stars whose equivalent widths were previously known, better agreement in equivalent widths was found if we used the excitation potentials and $\log g f$ values from Gurtovenko and Kostik (1982) for the Fe I lines.

On the other hand, excitation potentials from Moore et al. (1966) were taken for the Ba II lines. The $\log g f$ values used were those that reproduced the equivalent widths of the Ba II lines for the solar spectrum (Moore et al. 1966) when performing our spectral synthesis using the solar model (Kurucz 1979).

The spectral line synthesis was carried out point by point with a regular step of $0.01 \AA$. Only classical damping was considered, though the possible fluctuations in that parameter had a negligible effect compared with the equivalent widths uncertainties. The macroturbulent velocity was not important in the synthesis, as line profiles were of no interest.

Different equivalent widths obtained from different abundance values enabled the determination of the abundance for each spectral line; finally, the value of the abundance for each element was taken as the average of the individual results for each line.

The spectral synthesis program is able to reproduce the range of equivalent widths studied, even those higher than $200 \mathrm{m \AA}$; this has been checked comparing the results from the program with those from the literature on HR 774, (Tomkin and Lambert 1983) and Arcturus (Griffin 1968).

The remaining parameters affecting both the model atmosphere and the spectral lines studied, e.g., effective temperature, surface gravity, and microturbulent velocity, had a decisive importance in the equivalent width synthesis, so their determination needs to be discussed in more detail. The results for these three parameters are listed in Table IV.

\section{a) Effective Temperature}

The effective temperatures of the sample stars were calculated by the different calibrations with photometric indices. For this purpose, the color excess $\mathrm{E}(\boldsymbol{B}-V)$ needed to be determined. This was carried out on most stars by means of the method described by Janes (1977), which consists of an iterative process that converges to a fixed color excess value $\mathrm{E}(B-V)$, with an error less than $0.03 \mathrm{mag}$, allowing us to achieve the intrinsic indices $(B-V)_{0}, \mathrm{C}(45-48)_{0}$, $\mathrm{C}(42-45)_{0}$, and $\mathrm{C}(41-42)_{0}$. For those stars for which we could not apply this method, the one described by Janes and McClure (1975) was used.

TABLE II(a). Johnson colors.

\begin{tabular}{|c|c|c|c|c|c|c|c|c|c|}
\hline HD & & ame & $U-B$ & $B-V$ & V-R & $V-I$ & $\mathrm{~V}-\mathrm{J}$ & $V-K$ & $V-L$ \\
\hline \multicolumn{10}{|c|}{ Barium stars } \\
\hline 5395 & $\nu^{2}$ & Cas & 1.66 & 0.96 & 0.77 & 1.27 & & & \\
\hline 13520 & 60 & And & 3.22 & 1.48 & & & & & \\
\hline $\begin{array}{l}13611 \\
16458\end{array}$ & 65 & Cet & $\begin{array}{l}1.49 \\
1.30\end{array}$ & 0.88 & 0.67 & 1.16 & 1.51 & 2.06 & \\
\hline 30834 & 2 & Aur & 2.99 & 1.41 & 1.09 & 1.87 & & & \\
\hline 39003 & 32 & Aur & 2.23 & 1.14 & 0.82 & 1.38 & 1.79 & 2.46 & \\
\hline 43232 & $\gamma$ & Mon & 2.73 & 1.31 & 0.97 & 1.61 & 2.13 & 2.91 & \\
\hline 59294 & 6 & $\mathrm{CMi}$ & 2.66 & 1.29 & 0.90 & 1.54 & & & \\
\hline 69267 & $\beta$ & Cnc & 3.25 & 1.48 & 1.12 & 1.90 & 2.44 & 3.37 & 3.52 \\
\hline 74739 & j & Cnc & 1.81 & 1.03 & 0.75 & 1.24 & 1.56 & 2.14 & \\
\hline 82308 & $\lambda$ & Leo & 3.43 & 1.54 & 1.23 & 2.13 & 2.71 & 3.69 & 3.84 \\
\hline 83618 & 1 & Hya & 2.77 & 1.32 & 0.99 & 1.66 & 2.18 & 3.01 & 3.16 \\
\hline 95345 & 58 & Leo & 2.28 & 1.16 & & & & & \\
\hline 95578 & 61 & Leo & 3.54 & 1.62 & 1.33 & 2.29 & 2.90 & 3.92 & 4.08 \\
\hline 98262 & $v$ & UMa & 2.95 & 1.40 & 1.06 & 1.76 & 2.31 & 3.18 & 3.33 \\
\hline 98839 & 56 & UMa & 1.78 & 0.98 & 0.75 & 1.21 & 1.64 & 2.21 & \\
\hline 119228 & 83 & UMa & 3.59 & 1.63 & 1.40 & 2.52 & 3.19 & 4.25 & 4.43 \\
\hline 133208 & $\beta$ & Boo & 1.68 & 0.97 & 0.65 & 1.09 & 1.57 & 2.16 & \\
\hline 202109 & Zeta & Cyg & 1.75 & 0.99 & 0.70 & 1.18 & 1.55 & 2.11 & 2.22 \\
\hline 206778 & $\varepsilon$ & Peg & 3.22 & 1.52 & 1.05 & 1.81 & 2.36 & 3.20 & \\
\hline 218356 & 56 & Peg & 2.50 & 1.35 & 0.97 & 1.65 & & & \\
\hline \multicolumn{10}{|c|}{ Comparison stars } \\
\hline 3627 & $\delta$ & And & 2.76 & 1.28 & 0.92 & 1.58 & 2.04 & 2.80 & 2.98 \\
\hline $\begin{array}{l}12533 \\
27371\end{array}$ & $\begin{array}{r}57 \\
\gamma\end{array}$ & $\begin{array}{l}\text { And } \\
\text { Tau }\end{array}$ & $\begin{array}{l}2.13 \\
1.81\end{array}$ & $\begin{array}{l}1.21 \\
0.99\end{array}$ & 0.94 & 1.62 & 2.08 & 2.91 & 2.98 \\
\hline 28305 & $\varepsilon$ & Tau & 1.88 & 1.01 & 0.73 & 1.23 & 1.60 & 2.21 & \\
\hline 29139 & $\alpha$ & Tau & 3.46 & 1.54 & 1.23 & 2.17 & 2.70 & 3.67 & 3.86 \\
\hline $\begin{array}{l}62345 \\
67447\end{array}$ & K & Gem & $\begin{array}{l}1.62 \\
1.85\end{array}$ & $\begin{array}{l}0.92 \\
1.04\end{array}$ & 0.71 & 1.16 & 1.55 & 2.11 & \\
\hline $\begin{array}{l}76294 \\
77912\end{array}$ & 16 & Hya & $\begin{array}{l}1.82 \\
1.85\end{array}$ & $\begin{array}{l}1.00 \\
1.04\end{array}$ & $\begin{array}{l}0.71 \\
0.75\end{array}$ & $\begin{array}{l}1.20 \\
1.24\end{array}$ & 1.62 & 2.23 & 2.31 \\
\hline 124897 & $\alpha$ & Boo & 2.51 & 1.23 & 0.97 & 1.62 & 2.13 & 2.95 & 3.09 \\
\hline 129312 & 31 & Boo & 1.76 & 1.00 & & & & & \\
\hline 163770 & $\theta$ & Her & 2.81 & 1.35 & 0.90 & 1.53 & 2.04 & 2.84 & \\
\hline 205435 & م & Cyg & 1.45 & 0.89 & 0.71 & 1.21 & 1.47 & 2.05 & 2.14 \\
\hline 210807 & 24 & Cep & 1.53 & 0.92 & 0.69 & 1.17 & & & \\
\hline
\end{tabular}


TABLE II(b). DDO colors.

\begin{tabular}{|c|c|c|c|c|c|}
\hline HD & \multicolumn{2}{|c|}{ Name } & $C(45-48)$ & $C(42-45)$ & $C(41-42)$ \\
\hline \multicolumn{6}{|c|}{ Barium staŗs } \\
\hline 5395 & 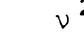 & ${ }^{2}$ Cas & 1.195 & 0.851 & 0.109 \\
\hline 13520 & 60 & And & 1.368 & 1.253 & 0.243 \\
\hline 13611 & 65 & Cet & & & \\
\hline 16458 & & & 1.341 & 1.033 & 0.455 \\
\hline 30834 & 2 & Aur & 1.317 & 1.101 & 0.257 \\
\hline 39003 & 32 & Aur & 1.234 & 0.938 & 0.292 \\
\hline 43232 & $\gamma$ & Mon & 1.304 & 1.027 & 0.314 \\
\hline 59294 & 6 & CMi & 1.292 & 1.022 & 0.326 \\
\hline 69267 & $\beta$ & $\mathrm{Cnc}$ & 1.373 & 1.263 & 0.254 \\
\hline 74739 & 1 & $\mathrm{Cnc}$ & 1.174 & 0.808 & 0.216 \\
\hline 82308 & $\lambda$ & Leo & & & \\
\hline 83618 & l & Hyа & 1.298 & 1.110 & 0.261 \\
\hline 95345 & 58 & Leo & 1.224 & 0.958 & 0.229 \\
\hline 95578 & 61 & Leo & & & \\
\hline 98262 & $\nu$ & UMa & 1.333 & 1.135 & 0.274 \\
\hline 98839 & 56 & UMa & 1.177 & 0.800 & 0.246 \\
\hline 119228 & 86 & UMa & & & \\
\hline 133208 & $\beta$ & Boo & 1.147 & 0.779 & 0.193 \\
\hline 202109 & Zeta & Cyg & 1.197 & 0.836 & 0.294 \\
\hline 206778 & $\varepsilon$ & Peg & 1.384 & 1.097 & 0.353 \\
\hline 218356 & 56 & Peg & & & \\
\hline \multicolumn{6}{|c|}{ Comparison stars } \\
\hline 3627 & $\delta$ & And & 1.301 & 1.118 & 0.336 \\
\hline 12533 & 57 & And & & & \\
\hline 27371 & $\gamma$ & Tau & 1.158 & 0.805 & 0.264 \\
\hline 28305 & $\varepsilon$ & Tau & 1.185 & 0.838 & 0.292 \\
\hline 29139 & $\alpha$ & Tau & & & \\
\hline 62345 & k & Gem & 1.165 & 0.805 & 0.200 \\
\hline 67447 & & & 1.178 & 0.790 & 0.249 \\
\hline 76294 & 16 & Hya & 1.177 & 0.822 & 0.237 \\
\hline 77912 & & & 1.187 & 0.805 & 0.246 \\
\hline 124897 & & Boo & 1.265 & 1.018 & 0.195 \\
\hline 129312 & 31 & Boo & 1.187 & 0.810 & 0.242 \\
\hline 163770 & $\theta$ & Her & 1.319 & 0.988 & 0.421 \\
\hline 205435 & $\rho$ & Cyg & 1.146 & 0.776 & 0.132 \\
\hline 210807 & 24 & Cep & 1.155 & 0.757 & 0.182 \\
\hline
\end{tabular}

TABLE III (a). Equivalent widths. Column 1: wavelength ( $\AA$ ); column 2: excitation potential (EV); column 3: oscillator strength; columns 4 10: equivalent width $(\mathrm{m} \AA$ ).

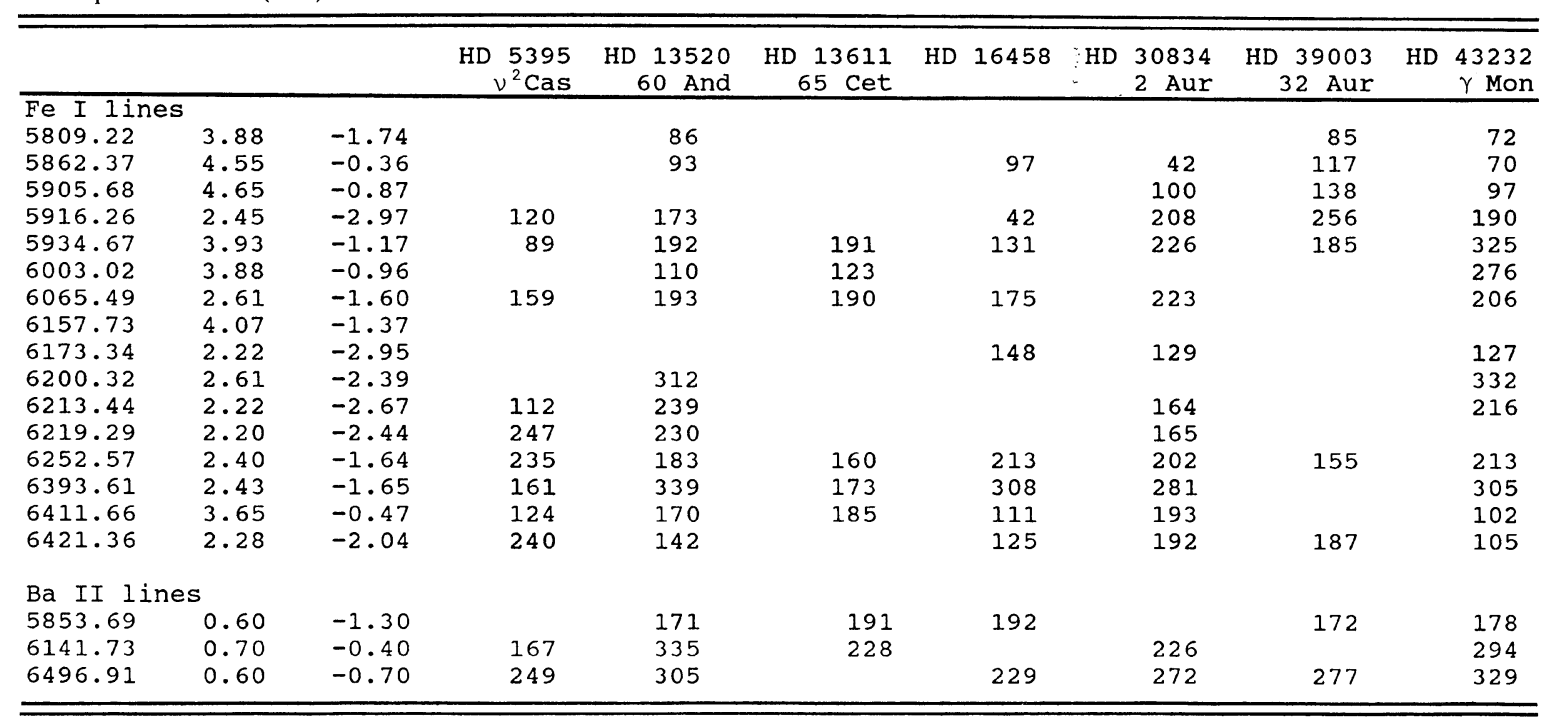


TABLE III(b). Equivalent widths (continued).

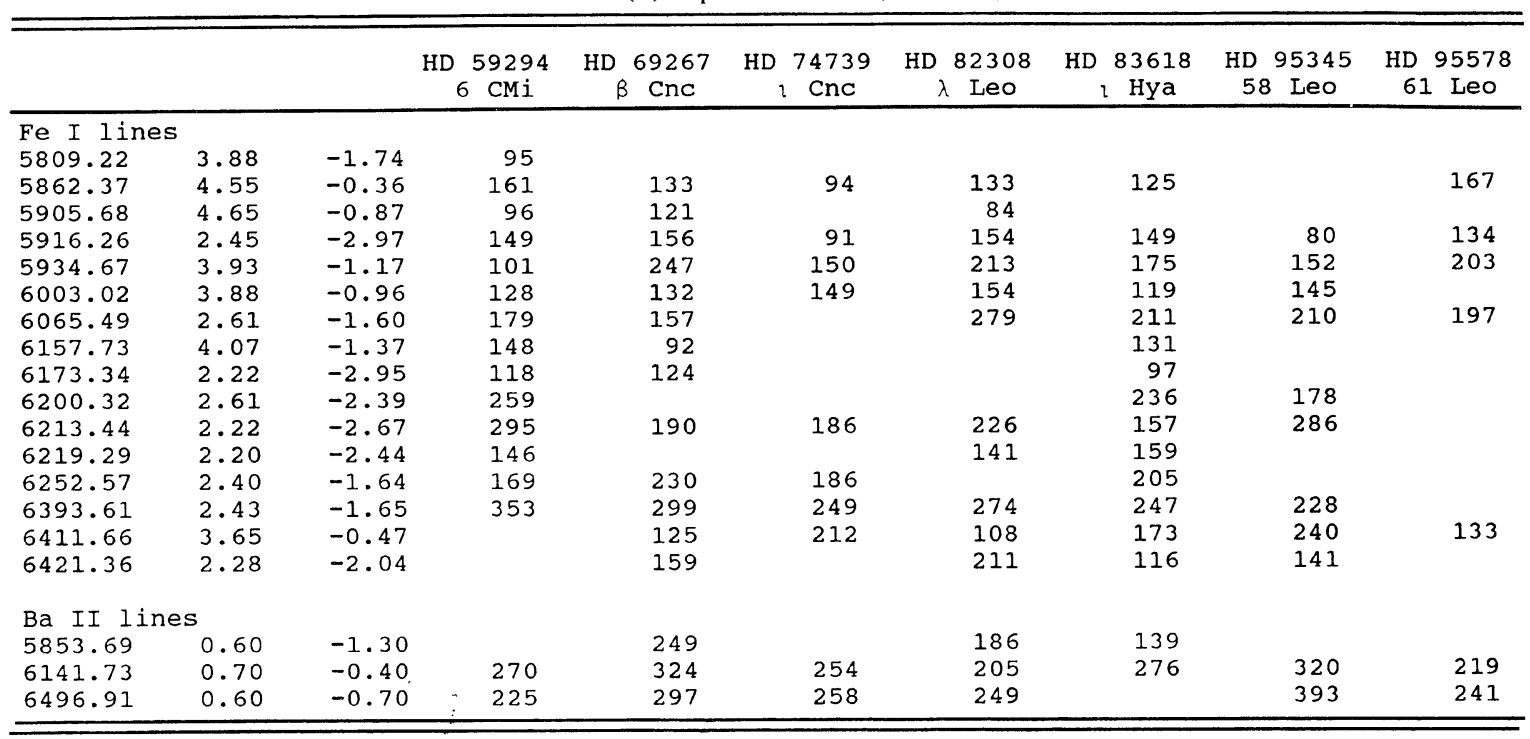

TABLE III (c). Equivalent widths (continued).

\begin{tabular}{|c|c|c|c|c|c|c|c|c|c|c|}
\hline & & & $\begin{array}{l}\mathrm{HD} g \\
\nu\end{array}$ & $\begin{array}{l}38262 \\
\text { UMa }\end{array}$ & $\begin{array}{c}\text { HD } 98839 \\
56 \mathrm{UMa}\end{array}$ & $\begin{array}{c}\mathrm{HD} 119228 \\
83 \mathrm{UMa}\end{array}$ & $\begin{array}{c}\text { HD } 133208 \\
\beta \text { Boo }\end{array}$ & $\begin{array}{l}\text { HD } 202109 \\
\text { Zeta Cyg }\end{array}$ & $\begin{array}{c}\text { HD } 206778 \\
\varepsilon \quad \text { Peg }\end{array}$ & $\begin{array}{c}\text { HD } 218356 \\
56 \text { Peg }\end{array}$ \\
\hline \multicolumn{11}{|c|}{ Fe I lines } \\
\hline 5809.22 & 3.88 & -1.74 & & 102 & & 143 & & 62 & 141 & 74 \\
\hline 5862.37 & 4.55 & -0.36 & & & 138 & 224 & & & 109 & \\
\hline 5905.68 & 4.65 & -0.87 & & & & & & 41 & 108 & \\
\hline 5916.26 & 2.45 & -2.97 & & 166 & 119 & & & 89 & 248 & 125 \\
\hline 5934.67 & 3.93 & -1.17 & & 218 & 237 & 219 & & 118 & 384 & 128 \\
\hline 6003.02 & 3.88 & -0.96 & & 137 & 135 & & 90 & 57 & 187 & \\
\hline 6065.49 & 2.61 & -1.60 & & 235 & 209 & 128 & 127 & 133 & 355 & 205 \\
\hline 6157.73 & 4.07 & -1.37 & & 108 & & & & & 115 & 203 \\
\hline 6173.34 & 2.22 & -2.95 & & 122 & & & 199 & 77 & 133 & 65 \\
\hline 6200.32 & 2.61 & -2.39 & & 337 & & & & & 347 & 389 \\
\hline 6213.44 & 2.22 & -2.67 & & & & 222 & 180 & 130 & 263 & 152 \\
\hline 6219.29 & 2.20 & -2.44 & & 189 & & 149 & & & 271 & \\
\hline 6252.57 & 2.40 & -1.64 & & 276 & & & & 175 & 259 & 155 \\
\hline 6393.61 & 2.43 & -1.65 & & 271 & 240 & 211 & 228 & 330 & 296 & 221 \\
\hline 6411.66 & 3.65 & -0.47 & & 122 & 151 & & 107 & 104 & 119 & 164 \\
\hline 6421.36 & 2.28 & -2.04 & & 131 & 210 & & 116 & 140 & 164 & \\
\hline \multicolumn{11}{|c|}{$\mathrm{Ba}$ II lines } \\
\hline 5853.69 & 0.60 & -1.30 & & 212 & 259 & 219 & & 117 & & 226 \\
\hline 6141.73 & 0.70 & -0.40 & & 231 & & 232 & 201 & 201 & 287 & 283 \\
\hline 6496.91 & 0.60 & -0.70 & & 250 & & & 254 & & 299 & \\
\hline
\end{tabular}

TABLE III(d). Equivalent widths (continued).

\begin{tabular}{|c|c|c|c|c|c|c|c|c|c|}
\hline & & & $\begin{array}{c}\text { HD } 3627 \\
\delta \text { And }\end{array}$ & $\begin{array}{c}\text { HD } 12533 \\
57 \text { And }\end{array}$ & $\begin{array}{c}\text { HD } 27371 \\
\gamma \text { Tau }\end{array}$ & $\begin{array}{c}\text { HD } 28305 \\
\varepsilon \mathrm{Tau}\end{array}$ & $\begin{array}{c}\text { HD } 29139 \\
\alpha \text { Tau }\end{array}$ & $\begin{array}{c}\text { HD } 62345 \\
\text { K Gem }\end{array}$ & HD $\quad 67447$ \\
\hline \multicolumn{10}{|c|}{ Fe I lines } \\
\hline 5809.22 & 3.88 & -1.74 & & 71 & & & 51 & & \\
\hline 5862.37 & 4.55 & -0.36 & 93 & 103 & & 64 & 114 & & \\
\hline 5905.68 & 4.65 & -0.87 & & 87 & & 115 & 68 & & 219 \\
\hline 5916.26 & 2.45 & -2.97 & 146 & 175 & 84 & 192 & 116 & 63 & \\
\hline 5934.67 & 3.93 & -1.17 & & 180 & 116 & 111 & 160 & 136 & \\
\hline 6003.02 & 3.88 & -0.96 & 78 & 116 & & & 123 & 67 & \\
\hline 6065.49 & 2.61 & -1.60 & 190 & 143 & 134 & 203 & 199 & & \\
\hline 6157.73 & 4.07 & -1.37 & 68 & 121 & & & 80 & 85 & 163 \\
\hline 6173.34 & 2.22 & -2.95 & 125 & 98 & & & 93 & 72 & \\
\hline 6200.32 & 2.61 & -2.39 & 332 & 282 & 371 & & 233 & & \\
\hline 6213.44 & 2.22 & -2.67 & & 374 & 149 & & 130 & & \\
\hline 6219.29 & 2.20 & -2.44 & & 258 & 159 & & 153 & & \\
\hline 6252.57 & 2.40 & -1.64 & 97 & 165 & 111 & & 164 & 138 & 127 \\
\hline 6393.61 & 2.43 & -1.65 & 174 & 233 & 195 & 212 & 224 & 204 & 277 \\
\hline 6411.66 & 3.65 & -0.47 & 130 & 94 & 112 & 151 & 106 & & 139 \\
\hline 6421.36 & 2.28 & -2.04 & 132 & 121 & & 131 & 116 & & 191 \\
\hline \multicolumn{10}{|c|}{$\mathrm{Ba}$ II lines } \\
\hline 5853.69 & 0.60 & -1.30 & & & & 103 & & & 197 \\
\hline 6141.73 & 0.70 & -0.40 & 110 & 200 & 274 & & 154 & 202 & 301 \\
\hline 6496.91 & 0.60 & -0.70 & & 233 & 264 & 264 & 233 & & 390 \\
\hline
\end{tabular}


TABLE III(e). Equivalent widths (continued)

\begin{tabular}{|c|c|c|c|c|c|c|c|c|c|c|c|c|}
\hline & & & $\begin{array}{c}\text { HD } 76294 \\
16 \text { Hya }\end{array}$ & HD & 77912 & HD & $\begin{array}{l}124897 \\
\alpha \text { BOO }\end{array}$ & $\begin{array}{c}\text { HD } 129312 \\
31 \text { Boo }\end{array}$ & $\begin{array}{c}\text { HD } 163770 \\
\theta \text { Her }\end{array}$ & HD & $\begin{array}{l}205435 \\
\rho \text { Cyg }\end{array}$ & $\begin{array}{c}\text { HD } 210807 \\
24 \text { Cep }\end{array}$ \\
\hline \multicolumn{13}{|c|}{$\mathrm{Fe}$ I lines } \\
\hline 5809.22 & 3.88 & -1.74 & 90 & & 174 & & 111 & 88 & & & & \\
\hline 5862.37 & 4.55 & -0.36 & & & 124 & & 50 & 178 & 76 & & & \\
\hline 5905.68 & 4.65 & -0.87 & 57 & & & & 82 & & & & & \\
\hline 5916.26 & 2.45 & -2.97 & & & 171 & & 111 & 263 & 190 & & 138 & \\
\hline 5934.67 & 3.93 & -1.17 & 88 & & & & 165 & & 222 & & & 71 \\
\hline 6003.02 & 3.88 & -0.96 & 114 & & 138 & & & 112 & 157 & & & 120 \\
\hline 6065.49 & 2.61 & -1.60 & 124 & & & & 121 & & 213 & & & \\
\hline 6157.73 & 4.07 & -1.37 & 136 & & 66 & & & & & & & \\
\hline 6173.34 & 2.22 & -2.95 & 30 & & & & 77 & & & & 124 & \\
\hline 6200.32 & 2.61 & -2.39 & 302 & & & & 187 & & 199 & & & \\
\hline 6213.44 & 2.22 & -2.67 & & & & & & 164 & 208 & & 111 & \\
\hline 6219.29 & 2.20 & -2.44 & & & & & 115 & & & & & \\
\hline 6252.57 & 2.40 & -1.64 & 250 & & 159 & & 160 & 128 & 231 & & 212 & 228 \\
\hline 6393.61 & 2.43 & -1.65 & 226 & & 205 & & 205 & 190 & & & 232 & \\
\hline 6411.66 & 3.65 & -0.47 & 111 & & & & & & & & & 169 \\
\hline 6421.36 & 2.28 & -2.04 & 163 & & 76 & & 122 & 129 & 144 & & & 180 \\
\hline \multicolumn{13}{|c|}{$\mathrm{Ba}$ II lines } \\
\hline 5853.69 & 0.60 & -1.30 & 121 & & 237 & & 109 & 187 & 237 & & & \\
\hline 6141.73 & 0.70 & -0.40 & & & & & 188 & 214 & 395 & & 194 & \\
\hline 6496.91 & 0.60 & -0.70 & & & & & 293 & & & & & 236 \\
\hline
\end{tabular}

TABLE IV. Physical parameters of the barium and comparison stars. $T_{\text {eff }}$ : effective temperature; $\log g$ : surface gravity; MICRO: microturbulent velocity $(\mathrm{km} / \mathrm{s})$.

\begin{tabular}{|c|c|c|c|c|}
\hline HD & Name & Teff & $\log g$ & MICRO \\
\hline \multicolumn{5}{|c|}{ Barium stars } \\
\hline 5395 & $v^{2}$ Cas & 4800 & 2.2 & 1.0 \\
\hline 13520 & 60 And & 3900 & 1.6 & 1.8 \\
\hline 13611 & 65 Cet & 5100 & 2.0 & 1.3 \\
\hline 16458 & & 4500 & 1.4 & 1.9 \\
\hline 30834 & 2 Aur & 4100 & 1.6 & 2.0 \\
\hline 39003 & 32 Aur & 4600 & 2.0 & 1.8 \\
\hline 43232 & $\gamma$ Mon & 4400 & 1.6 & 1.2 \\
\hline 59294 & $6 \mathrm{CMi}$ & 4400 & 1.8 & 1.6 \\
\hline 69267 & B Cnc & 3900 & 1.6 & 1.0 \\
\hline 74739 & I Cnc & 4900 & 2.2 & 1.6 \\
\hline 82308 & $\lambda$ Leo & 3900 & 1.6 & 1.8 \\
\hline 83618 & I Hya & 4200 & 1.8 & 1.1 \\
\hline 95345 & 58 Leo & 4500 & 2.2 & 2.3 \\
\hline 95578 & 61 Leo & 3700 & 1.4 & 1.6 \\
\hline 98262 & v UMa & 4100 & 1.6 & 1.5 \\
\hline 98839 & $56 \mathrm{UMa}$ & 4900 & 2.0 & 1.6 \\
\hline 119228 & $83 \mathrm{UMa}$ & 3600 & 1.6 & 1.8 \\
\hline 133208 & B Boo & 4900 & 2.8 & 1.5 \\
\hline 202109 & Zeta Cyg & 4900 & 2.0 & 1.4 \\
\hline 206778 & $\varepsilon$ Peg & 4400 & 1.2 & 2.3 \\
\hline 218356 & 56 Peg & 5100 & 1.2 & 1.7 \\
\hline \multicolumn{5}{|c|}{ Comparison stars } \\
\hline 3627 & $\delta$ And & 4200 & 2.0 & 1.2 \\
\hline 12533 & 57 And & 4300 & 1.4 & 1.6 \\
\hline 27371 & $\gamma$ Tau & 4800 & 2.6 & 1.3 \\
\hline 28305 & $\varepsilon \mathrm{Tau}$ & 4800 & 2.2 & 2.1 \\
\hline 29139 & $\alpha \mathrm{Tau}$ & 3800 & 1.8 & 1.0 \\
\hline 62345 & K Gem & 4900 & 2.4 & 1.1 \\
\hline 67447 & & 5000 & 2.0 & 1.0 \\
\hline 76294 & 16 нуа & 4900 & 2.2 & 1.2 \\
\hline 77912 & & 5000 & 2.0 & 1.1 \\
\hline 124897 & $\alpha$ Boo & 4300 & 2.0 & 1.5 \\
\hline 129312 & 31 Boo & 4800 & 1.8 & 1.3 \\
\hline 163770 & $\theta$ Her & 4700 & 1.4 & 1.6 \\
\hline 205435 & $\rho \quad$ cyg & 4900 & 2.8 & 2.3 \\
\hline 210807 & 24 Cep & 5000 & 2.2 & 2.6 \\
\hline
\end{tabular}

Lü and Sawyer (1979) have obtained spectral energy distributions for some barium stars which exhibit a conspicuous absorption feature near $4100 \AA$ due to the Bond-Neff depression. For wavelengths greater than $4200 \AA$ this effect is not appreciable. Therefore, the DDO bands $m_{42}-m_{48}$ and the $\mathrm{E}(B-V)$, computed using the method of Janes (1975), are substantially free of such an effect. In any case, the uncertainties produced by a possible influence of the Bond-Neff depression will be less than the errors of the temperature calibration $T_{\text {eff }}$ vs $(B-V)$.

As other photometric calibrations were performed, we were to determine the color excesses corresponding to the photometric indices used for that purpose. This was carried out by using the interstellar reddening curve from Savage and Mathis (1979) and comparing the results with those obtained from Johnson (1966) using the Van de Hulst curve No. 15.

The calibrations used were those from Ridgway et al. (1980), with the intrinsic index $(V-K)_{0}$; Böhm-Vitense $(1981)$, with the $(B-V)_{0}$ index for giant and supergiant stars with solar metallicity (the uncertainties are $250 \mathrm{~K}$ ); and Johnson (1968), using the relations among effective temperature and photometric indices $U-V, B-V, V-R$, $V-I, V-J, V-K$, and $V-L$ and spectral type versus effective temperature.

Finally, we adopted the mean value from the aforementioned calibrations as the effective temperature of each star.

\section{b) Surface Gravity}

Log $g$ values were obtained from Janes and McClure (1975) using DDO photometry indices $C(45-48)$ and $\mathrm{C}(42-45)$, which were previously corrected for extinction as explained above. The results agreed with those from Morel et al. (1976) and Williams (1975) for stars having the same luminosity class.

As our sample contains mainly weak barium stars, the differences in the DDO indices resulting from peculiarity do not exceed the error inherent in the Janes and McClure cali- 
bration of $\log g$. This can be checked taking into account the mean magnitude differences of Lü and Sawyer (1979) for the DDO bands between standard and barium stars. For the strongest barium star in the sample, HR 774, with a Warner index of 3 , the mean differences in the DDO indices lead us to an error of 0.2 in $\log g$, which is no larger than that resulting from the calibration.

\section{c) Microturbulent Velocity}

The microturbulent velocity was determined using the set of Fe I lines listed in Tables III(a)-III(b), with the Zeta Cap empirical curve of growth for Fe I (Tech 1971).

\section{RESULTS AND DISCUSSION}

Once all of the previous parameters were fixed and model atmospheres assigned for each individual star, the above procedures were followed to carry out spectral synthesis, leading to the determination of iron and barium abundances. The results concerning the abundance of the whole sample of stars are shown in Table V, where a distinction between the comparison and peculiar barium stars has been made. As the number of lines on which the abundance determinations were based is not high enough, we adopted the confidence limit given by the Student's $t$ distribution as the abundance error; when the number of lines was two, the minimum and maximum values were taken. The results concerning iron and barium abundances, as well as the confidence limits, are shown in Fig. 1.

In Fig. 2 the iron abundances obtained in this paper have been plotted versus those from Morel et al. (1976) and Williams (1975) for the stars in common. The values range from $[\mathrm{Fe} / \mathrm{H}]=-1.00$ for $\mathrm{HD} 5395$ (while Williams gives -0.75 ) to $[\mathrm{Fe} / \mathrm{H}]=0.55$ for $\alpha$ Boo (while Williams gives 0.25 ); the latter is based on the synthesis of only a single Fe I line.

In addition, Pilachowski (1977) gives $[\mathrm{Fe} / \mathrm{H}]=-0.50$ for $\mathrm{HR} 774$ while our value is $[\mathrm{Fe} / \mathrm{H}]=-0.36$, $[\mathrm{Fe} / \mathrm{H}]=-0.20$ for $\beta$ Cnc while ours is $[\mathrm{Fe} / \mathrm{H}]=-0.07$, and $[\mathrm{Fe} / \mathrm{H}]=-0.10$ for Zeta Cyg compared with our value of $[\mathrm{Fe} / \mathrm{H}]=-0.17$. Cowley (1968) gives $[\mathrm{Fe} / \mathrm{H}]=-0.18$ for $\mathrm{HR} 774$ while the value

TABLE V. Iron and barium abundances. Column 1: HD number; column 2: star name; columns 35: iron abundance, confidence limit, and number of $\mathrm{Fe}$ I lines; columns 6-8: barium abundance, confidence limit, and number of $\mathrm{Ba}$ II lines; column 9: barium-over-iron excess.

\begin{tabular}{|c|c|c|c|c|c|c|c|c|c|}
\hline HD & & Name & {$[\mathrm{Fe} / \mathrm{H}]$} & C.L. & N. & {$[\mathrm{Ba} / \mathrm{H}]$} & C.L. & $\mathrm{N}$. & {$[\mathrm{Ba} / \mathrm{Fe}]$} \\
\hline \multicolumn{10}{|c|}{ Barium stars } \\
\hline 5395 & & $v^{2}$ Cas & -1.00 & 0.10 & 2 & 1.00 & 0.20 & 2 & 2.00 \\
\hline 13520 & 60 & And & 0.30 & & 1 & 0.88 & & 1 & 0.58 \\
\hline 13611 & & Cet & -0.01 & 0.05 & $\overline{2}$ & 0.30 & 0.20 & 2 & 0.31 \\
\hline 16458 & & & -0.36 & 0.07 & 6 & 0.60 & 0.10 & 2 & 0.96 \\
\hline 30834 & 2 & Aur & -0.05 & 0.31 & 3 & 0.69 & & 1 & 0.74 \\
\hline 39003 & 32 & Aur & -0.12 & & 1 & -0.10 & & 1 & 0.02 \\
\hline 43232 & & Y Mon & 0.04 & 0.36 & 6 & 0.75 & 0.05 & 2 & 0.71 \\
\hline 59294 & & $\mathrm{CMi}$ & 0.12 & 0.27 & 4 & 1.10 & & 1 & 0.98 \\
\hline 69267 & & B Cnc & -0.05 & 0.29 & 5 & 0.77 & 0.72 & 3 & 0.82 \\
\hline 74739 & & i Cnc & -0.07 & 0.13 & 6 & 0.37 & 0.21 & 2 & 0.44 \\
\hline 82308 & & $\lambda$ Leo & 0.05 & 0.15 & 5 & 0.97 & 0.08 & 2 & 0.92 \\
\hline 83618 & & i Hya & 0.05 & 0.23 & 5 & 0.80 & & 1 & 0.75 \\
\hline 95345 & 58 & 3 Leo & -0.18 & 0.22 & 6 & 0.75 & 0.05 & 2 & 0.93 \\
\hline 95578 & 61 & Leo & -0.23 & 0.04 & 2 & 0.27 & & 1 & 0.50 \\
\hline 98262 & & UMa & -0.02 & 0.16 & 5 & 0.25 & 0.05 & 2 & 0.27 \\
\hline 98839 & 56 & UMa & 0.23 & 0.15 & 6 & 0.56 & & 1 & 0.33 \\
\hline 119228 & 83 & UMa & 0.30 & & 1 & 0.30 & & 1 & 0.00 \\
\hline 133208 & & B Boo & 0.55 & & 1 & 0.81 & 0.10 & 2 & 0.26 \\
\hline 202109 & Zeta & Cyg & -0.17 & 0.31 & 5 & 0.40 & & 1 & 0.57 \\
\hline 206778 & & $\varepsilon$ Peg & 0.04 & 0.22 & 6 & 0.60 & & 1 & 0.56 \\
\hline 218356 & 56 & Peg & -0.15 & 0.43 & 3 & 0.94 & 0.02 & 2 & 1.09 \\
\hline \multicolumn{10}{|c|}{ Comparison stars } \\
\hline 3627 & $\delta$ & $\delta$ And & 0.46 & & 1 & -0.69 & & 1 & $-1 \cdot 15$ \\
\hline 12533 & 57 & And & 0.09 & 0.50 & 5 & 0.20 & & 1 & 0.11 \\
\hline 27371 & & y Tau & 0.09 & 0.15 & 4 & 0.60 & & 1 & 0.51 \\
\hline 28305 & & $\varepsilon \mathrm{Tau}$ & -0.04 & 0.12 & 2 & 0.44 & & 1 & 0.48 \\
\hline 29139 & & x Tau & -0.17 & 0.14 & 7 & -0.06 & 0.01 & 2 & 0.11 \\
\hline 62345 & & K Gem & 0.23 & 0.06 & 2 & 0.69 & & 1 & 0.46 \\
\hline 67447 & & & -0.30 & & 1 & 1.31 & 0.25 & 3 & 1.61 \\
\hline 76294 & 16 & Hya & -0.21 & 0.40 & 3 & -0.19 & & 1 & 0.02 \\
\hline 77912 & & & 0.38 & 0.26 & 3 & 1.31 & & 1 & 0.93 \\
\hline 124897 & $\alpha$ & $\alpha$ Boo & -0.69 & 0.10 & 5 & -0.40 & & 1 & 0.29 \\
\hline 129312 & 31 & Boo & -0.44 & & 1 & 0.70 & & 1 & 1.14 \\
\hline 163770 & $\theta$ & $\theta$ Her & 0.25 & 0.40 & 4 & 1.30 & & 1 & 1.05 \\
\hline 205435 & م & Cyg & -0.05 & 0.25 & 2 & 0.56 & & 1 & 0.61 \\
\hline 210807 & 24 & Cep & -0.14 & & 1 & 0.20 & & 1 & 0.34 \\
\hline
\end{tabular}


HD

Name

$[\mathrm{Fe} / \mathrm{H}]$

[Ba/H]

Barium

13520

13611

16458

30834

39003

43232

59294

69267

74739

82308

83618

95345

95578

98262

98839

119228

133208

202109

206778

218356

Comparison stars 3627

12533

27371

28305

29139

62345

67447

76294

77912

124897

129312

163770

205435

210807
$-1.2-0.8-0.4+0.0+0.4+0.8-0.6-0.2+0.2+0.6+1.0+1.4$

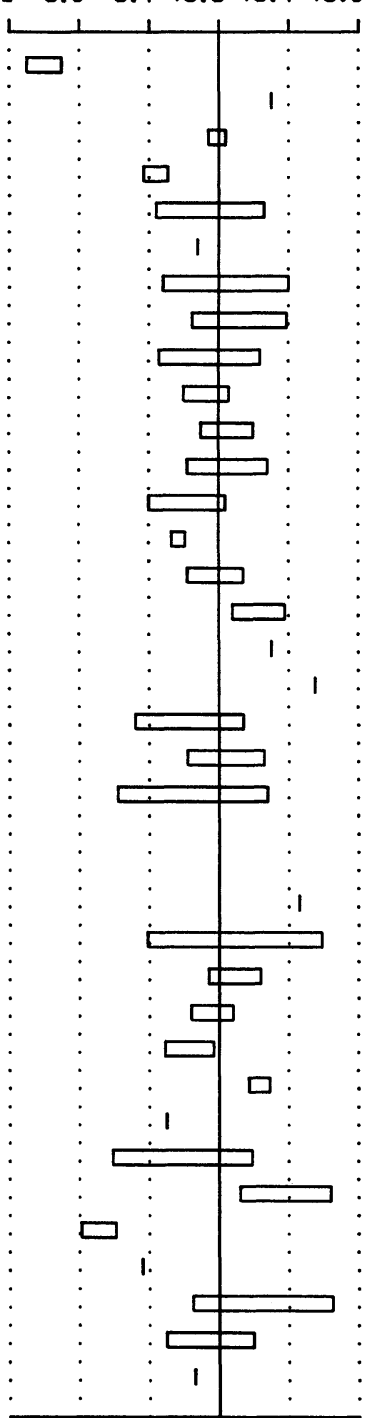

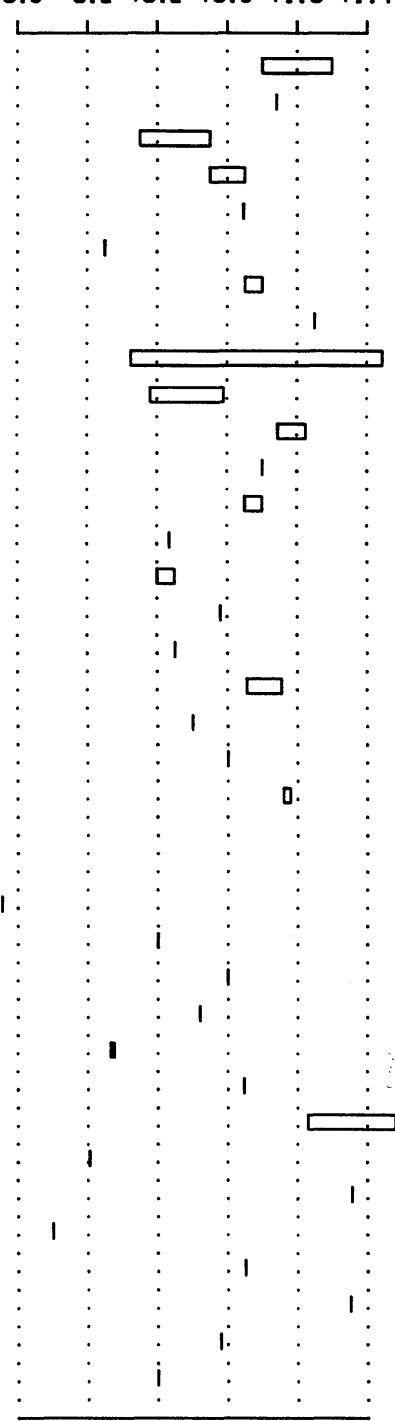

FIG. 1. Iron and barium abundances for the sample of barium and comparison stars. Confidence limits are shown when possible. 


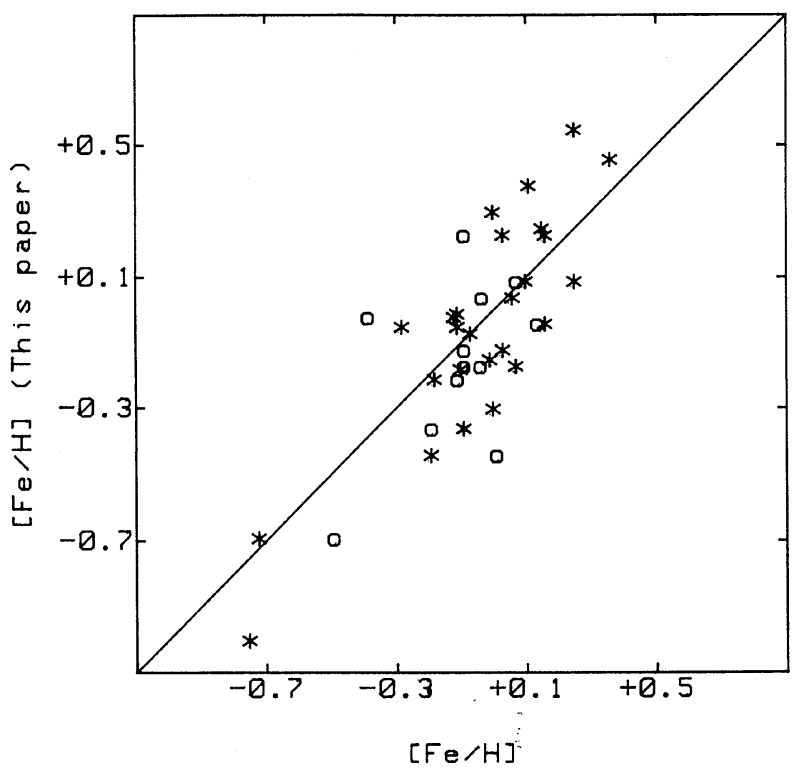

FIG. 2. Iron abundances from Morel et al. (1976) (O) and Williams $(1975)(*)$, vs the values in this paper.

from Tomkin and Lambert $(1983)$ is $[\mathrm{Fe} / \mathrm{H}]=-0.43$.

The barium abundances from this paper have been plotted versus the ones from Williams (1975) (Fig. 3). The barium group has barium abundances higher than the solar value; 6 $\mathrm{CMi}$ is an outstanding case, with $[\mathrm{Ba} / \mathrm{H}]=1.1$, although this result is based on only one Fe I line. The exception is 32 Aur, with $[\mathrm{Ba} / \mathrm{H}]=-0.10$. The comparison stars have barium abundances distributed around the solar value, with $[\mathrm{Ba} / \mathrm{H}]=-0.69$ for Delta And as the lowest one. Extreme cases are HD 67447 and HD 77912, each with barium abundances $[\mathrm{Ba} / \mathrm{H}]=1.31$, higher than any of the barium stars.

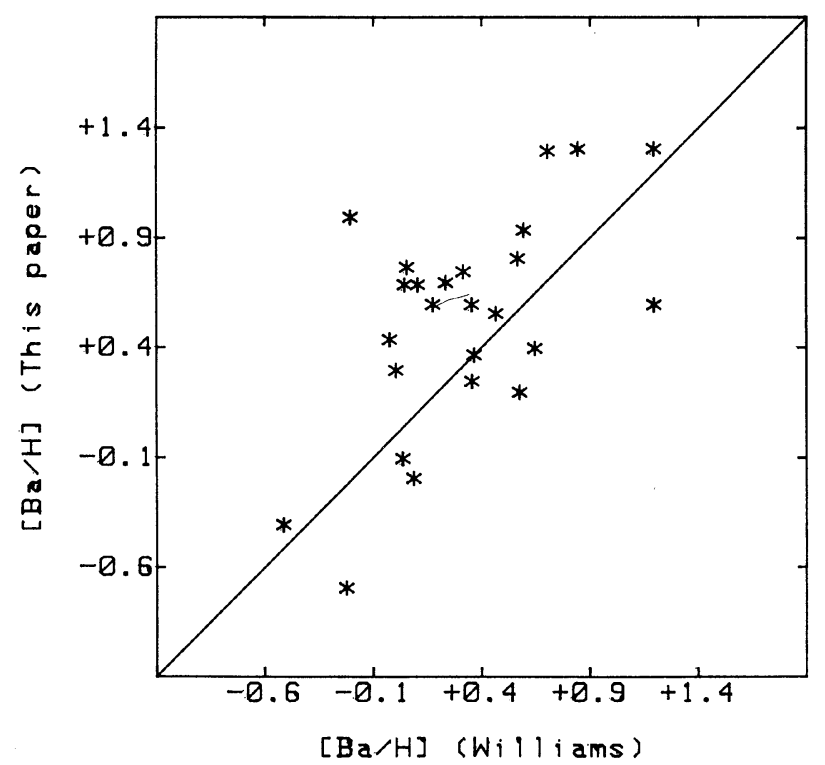

FIG. 3. Barium abundances from Williams (1975) vs the values in this paper.

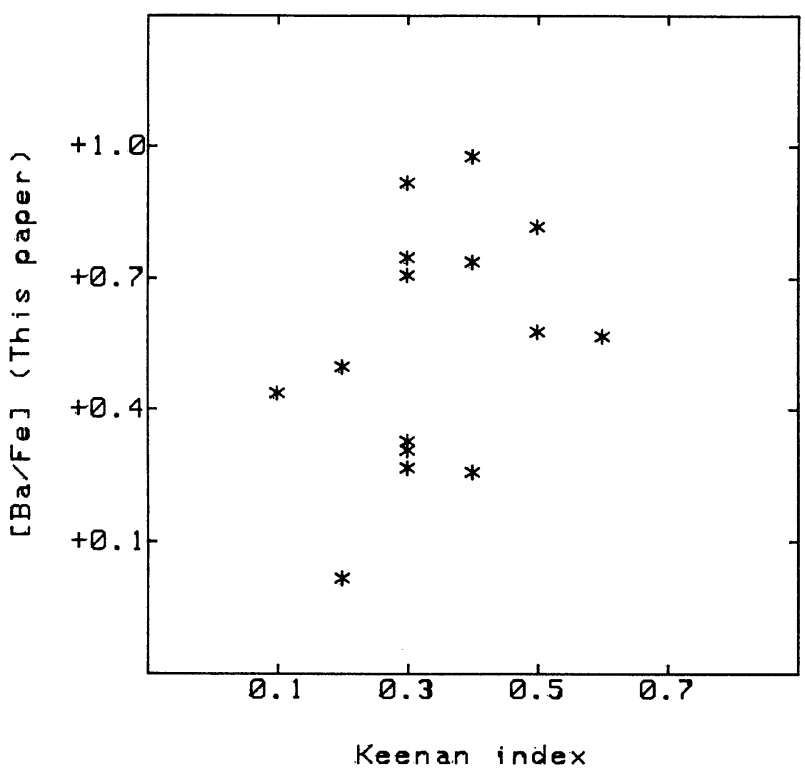

FIG. 4. Barium-over-iron excess vs Keenan barium indicator for the sample of barium stars studied.

Pilachowski (1977) gives a value of $[\mathrm{Ba} / \mathrm{H}]=1.0$ for HR 774, while the abundance from Tomkin and Lambert $(1983)$ is $[\mathrm{Ba} / \mathrm{H}]=0.82$, that from Cowley (1968) is $[\mathrm{Ba} / \mathrm{H}]=0.80$ and our result is $[\mathrm{Ba} / \mathrm{H}]=0.60$. Zeta Cyg and $\beta$ Cnc have barium abundances $[\mathrm{Ba} / \mathrm{H}]=0.30$ and 0.20 , respectively, while our results are $[\mathrm{Ba} / \mathrm{H}]=0.40$ and 0.37 .

We have used the excess of barium over iron, $[\mathrm{Ba} / \mathrm{Fe}]$, as the most representative indicator of peculiarity. These values, shown in Table V, should be compared with the Keenan index (Fig. 4); that there is no correlation may be due to the dependence of the Keenan indicator on the temperature and

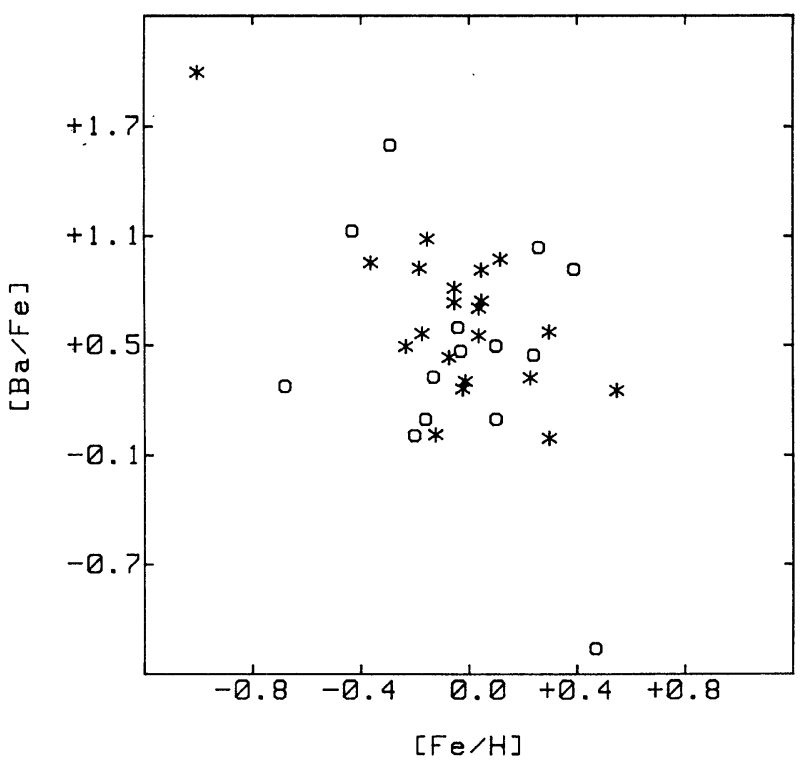

FIG. 5. Barium-over-iron excess vs iron abundance for comparison $(\mathrm{O})$ and barium $(*)$ stars. 
surface gravity.

There is not a definite difference in $[\mathrm{Ba} / \mathrm{Fe}]$ between the barium and the comparison stars in the sample studied because the barium stars selected are not very peculiar. In this sense, there are comparison stars with barium-over-iron excess higher than those corresponding to barium stars. Among them, HD 67447 stands out, with $[\mathrm{Ba} / \mathrm{Fe}]=1.61$, perhaps a new barium star. On the other hand, Delta And, with $[\mathrm{Ba} / \mathrm{Fe}]=-1.15$, represents the least peculiar star in the sample. The star showing the highest barium-over-iron excess, $[\mathrm{Ba} / \mathrm{Fe}]=2.00$, is $v^{2} \mathrm{Cas}$, which has a barium abundance $[\mathrm{Ba} / \mathrm{H}]=1.00$. This is in the opposite sense to the value given by Williams (1975), $[\mathrm{Ba} / \mathrm{H}]=-0.20$, for a peculiar star with a Warner index of 2.

In Fig. 5 we have plotted the barium-over-iron excess $[\mathrm{Ba} / \mathrm{Fe}]$ vs iron abundance, trying to confirm the correlation pointed out by Kovacs (1985), in the sense that the lower the iron content, the more overabundant barium appears to be relative to iron. If this is so, "barium strong" stars would be older than "barium weak" ones, in agreement with
Catchpole et al. (1977), who found a higher velocity dispersion for the "barium strong" group. Unfortunately, most of the stars in our sample exhibit an iron content in the range $-0.5 \leqslant[\mathrm{Fe} / \mathrm{H}] \leqslant 0.5$; only in one case is this value roughly - 1.0, giving rise to a significant lack of data in the $-1.0 \leqslant[\mathrm{Fe} / \mathrm{H}] \leqslant-0.5$ range. Therefore, very little can be predicted about the existence of an anticorrelation. However, if our data and those of Kovacs (1985) are plotted together, a general trend can be observed. If such an anticorrelation is even doubtful, the differences in velocity dispersion between both groups could be explained by assuming the hypothesis that these stars are all members of binary systems. Concerning this, McClure (1983) has studied the variations in radial velocities, finding them lower for the "barium weak" group, which would indicate that these systems are bound in orbits with larger radii than those belonging to the "strong" group. In this way, the transfer of heavy elements from one member of the binary system to the other would be smaller for the "barium weak" group (Böhm-Vitense et al. 1984), leading to a lower barium overabundance.

\section{REFERENCES}

Bidelman, W. P., and Keenan, P. C. (1951). Astrophys. J. 114, 473 Böhm-Vitense, E. (1981). Annu. Rev. Astron. Astrophys. 19, 314.

Bohm-Vitense, E., Nemec, J., and Proffitt, C. (1984). Astrophys. J. 278, 726.

Catchpole, R. M., Robertson, B. S. C., and Warren, P. R. (1977). Mon. Not. R. Astron. Soc. 181, 391.

Cornide, M., and Rego, M. (1985). Astrophys. Space Sci. 105, 55. Cowley, C. R. (1968). Astrophys. J. 153, 169.

Griffin, R. F. (1968). A Photometric Atlas of the Spectrum of Arcturus (Cambridge Philosophical Society, Cambridge, England).

Gustafsson, B. (1984). Private communication.

Gurtovenko, E. A., and Kostik, R. I. (1982). Astron. Astrophys. Suppl. 47, 193.

Hoffleit, D., and Jaschek, C. (1982). Catalogue of Bright Stars (Yale University, New Haven ).

Janes, K. A., and McClure, R. D. (1975). Dudley Obs. Rep. 9, 99.

Janes, K. A. (1977). Publ. Astron. Soc. Pac. 89, 576.

Johnson, H. L. (1966). Annu. Rev. Astron. Astrophys. 4, 193.

Johnson, H. L. (1968). in Stars and Stellar Systems, edited by B. M. Middlehurst and L. H. Aller (University of Chicago, Chicago), Vol. VII, p. 167.

Keenan, P. C. (1983). Centre de Donnés Stellaires Bull. 24, 19.

Keenan, P. C. (1984). News. Chem. Peculiar Late-Type Stars 1, 5.

Keenan, P. C. (1985). CDS Bull. 29, 25.

Kovacs, N. (1983). Astron. Astrophys. 124, 63.

Kovacs, N. (1985). Astron. Astrophys. 150, 232.

Kurucz, R. L. (1979). Astrophys. J. Suppl. 40, 1.
Lü, P. K., Dawson, D. W., Upgren, A. R., and Weis, E. W. (1983). Astrophys. J. Suppl. 52, 169.

Lü, P. K., and Sawyer, D. (1979). Astrophys. J. 231, 144

McClure, R. D. (1976). Astron. J. 81, 182.

McClure, R. D., and Forrester, W. T. (1981). Publ. Dominion Astrophys. Obs. XV, No. 14.

McClure, R. D. (1983). Astrophys. J. 268, 264.

McClure, R. D. (1984). Publ. Astron. Soc. Pac. 96, 117.

McConnell, D. J., Frye, R. L., and Upgren, A. R. (1972). Astron. J. 77, 384.

Moore, C. E., Minnaert, M. G. J., and Houtgast, J. (1966). The Solar Spectrum 2935 A to 8770 A, NBS Monograph No. 61 (U. S. Government Printing Office, Washington, DC).

Morel, M., Bentolila, C., Cayrel, G., and Hauck, B. (1976). In Abundance Effects in Classification, IAU Symposium No. 72, edited by B. Hauck and P. C. Keenan (Reidel, Dordrecht).

Pilachowski, C. A. (1977). Astron. Astrophys. 54, 465.

Ridgway, S. T., Joyce, R. R., White, N. M., and Wing, R. F. (1980). Astrophys. J. 235, 126.

Savage, B. D., and Mathis, J. S. (1979). Annu. Rev. Astron. Astrophys. 17, 73.

Tech, J. L. (1971) . A High-Dispersion Spectral Analysis of the Ba II Star HD 204075, NBS Monograph No. 119, (NBS, Washington, DC).

Tomkin, J., and Lambert, D. L. (1983). Astrophys. J 273, 722.

Warner, B. (1965). Mon. Not. R. Astron. Soc. 129, 263.

Williams, P. M. (1975). Mon. Not. R. Astron. Soc. 170, 343. 